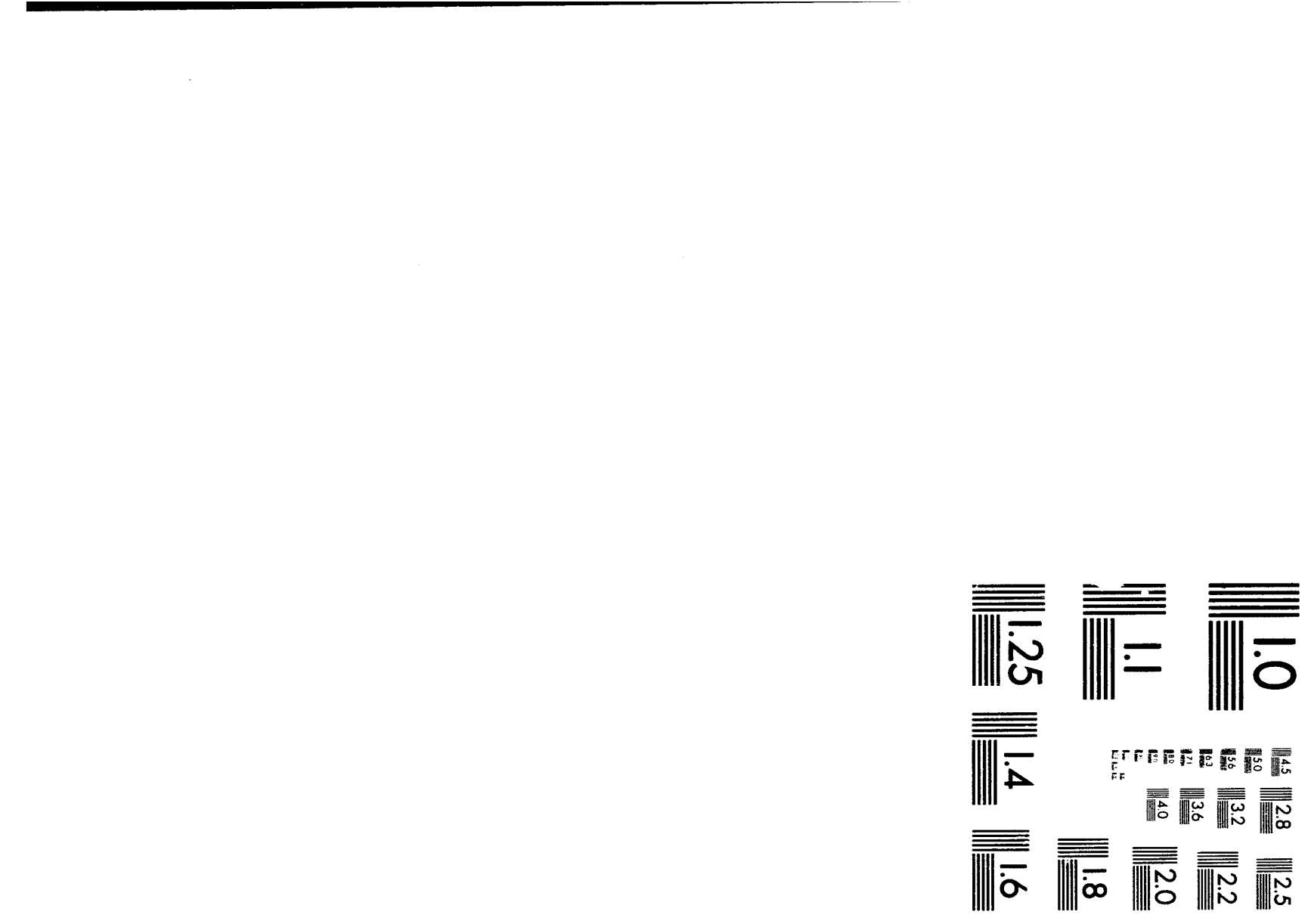$$
5
$$

.



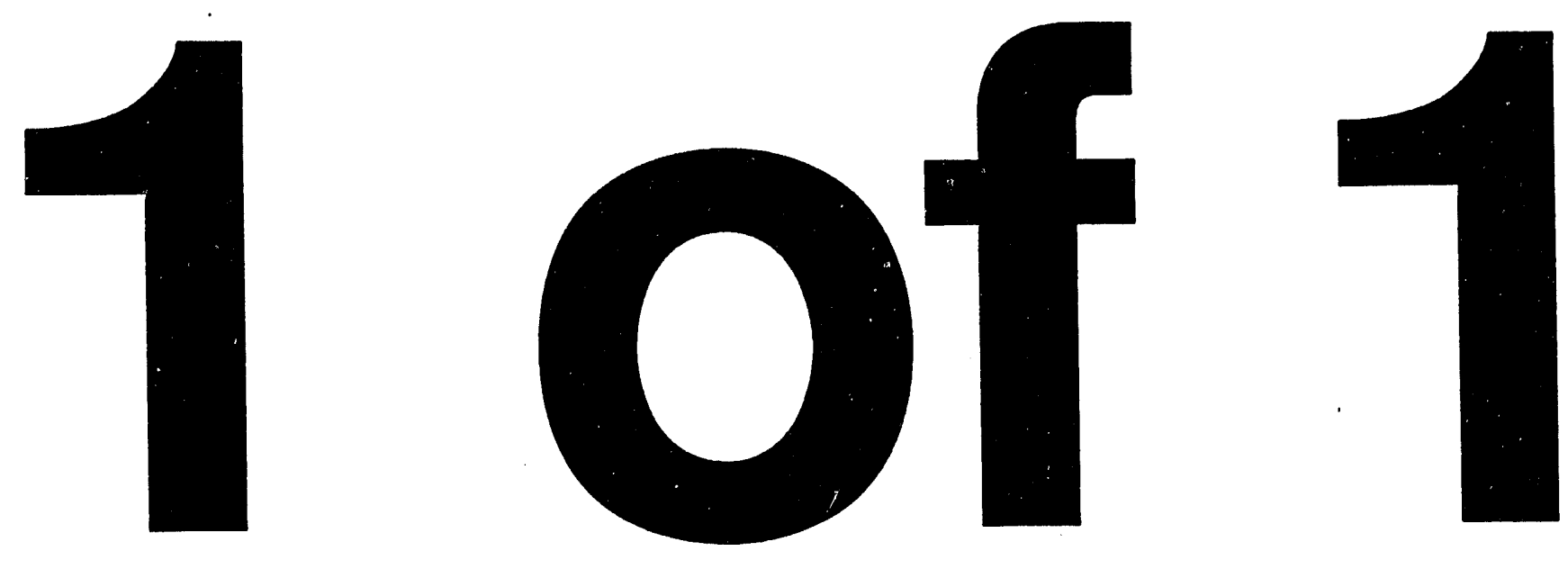
TITLE: Genetic Algorithms for Minimal Source Reconstructions

AUTHOR(S): Paul S. Lewis, John C. Mosher

Proc. 27th. Ann. Asilomar Conf. on Signals, Systems and Computers, Pacific Grove, CA Nov. 1-3, 1.993

\section{DISCLAIMER}

This report was prepared as an account of work sponsored by an agency of the United States Government. Neither the United States Government nor any agency thereof, nor any of their employees, makes any warranty, express or implied, or assumes any legal liability or responsibility for the accuracy, completeness, or usefulness of any information, apparatus, product, or process disclosed, or represents that its use would not infringe privately owned rights. Reference herein to any specific commercial product, process, or service by trade name, trademark, manufacturer, or otherwise does not necessarily constitute or imply its endorsement, recommendation, or favoring by the United States Government or any agency thereof. The views and opinions of authors expressed herein do not necessarily state or reflect those of the United States Government or any agency thereof. 
To appear in: Proc. Twenty-Seventh Ann. Asilomar Conf. on Signals, Sys-

tems, and Computers, Pacfic Crove, CA, Nov. 1-3, 1993.

\title{
Genetic Algorithms for Minimal Source Reconstructions
}

\author{
Paul S. Lewis and John C. Mosher \\ Los Alamos National Laboratory \\ Group ESA-6, MS J580 \\ Los Alamos NM, 87545 USA \\ email: lewis@lanl.gov
}

\begin{abstract}
Under-determined linear inverse problems arise in applications in which signals must be estimated from insufficient data. In these problems the number of potentially active sources is greater than the number of observations. In many situations, it is desirable to find a minimal source solution. This can be accomplished by minimizing a cost function that accounts for both the compatibility of the solution with the observations and for its "sparseness." Minimizing functions of this form can be a difficult optimization problem. Genetic algorithms are a relatively new and robust approach to the solution of difficult optimization problems, providing a global framework that is not dependent on local continuity or on explicit starting values. In this paper, we describe the use of genetic algorithms to find minimal source solutions, using as an example a simulation inspired by the reconstruction of neural currents in the human brain from magnetoencephalographic (MEG) measurements.
\end{abstract}

\subsection{Introduction}

Underdetermined linear inverse problems [1] arise in applications in which signals must be estimated from insufficient data. Examples occur in image enhancement, tomographic reconstruction, filter and array design, spectral estimation, and system modeling. A common characteristic is that the observed signals are due to the superposition of individual sources, and that the number of potentially active sources is greater than the number of observations. The form of the forward problem can be expressed as $y=A x$, where the dimension of the source vector $x$ is far greater than the rank of $\boldsymbol{A}$. As a consequence, there is no mathematically unique inverse-many different source configurations can produce the same observations. In this situation solution of the inverse involves finding a source configuration that is not only consistent with the observations, but that also optimizes

Los Alamos National Laboratory is operated by the University of California for the United States Department of Energy under contract W7405-ENG-36. some other criteria. For example, the minimum norm solution is the shortest length $x$ that satisfies the equation.

In many cases, it is desirable to find a solution with the minimum number of activ e sources. In signal reconstruction applications, a minimal source solution may be motivated by the a priori knowledge that the sources are sparse and highly localized. In design applications a minimal source solution may be motivated by the desire to minimize hardware elements while siill meeting performance specifications. One approach to finding a minimal source solution is to minimize a cost function that accounts both for the compatibility of the solution with the observations and for its "sparseness." This function may be derived empirically or through a statistical analysis, e.g., maximum a posteriori estimation. The resulting function is not likely to be well behaved. In fact, it will generally be multimodal and can often be discontinuous. The result is a difficult optimization problem.

Genetic algorithms $[2,3]$ are a relatively rew and robust approach to the solution of difficult aptimization problems. Genetic algorithms provide a global frumework for optimization that does not depend on local continuity or on explicit starting values. In this vaper. we tescribe the use of genetic algorithmis is find minimal source colutions. We illustrate the application, of these algorithrse, with a simulation example inspire ' hy $\mathrm{d}$. problem of neuromagnetic source reconstructio:

\subsection{Algorithms}

A genetic algorithm wo $\mathrm{ks}$ in a topulation of strings defined over a finite as $\rho^{\prime}$ anc: By process of reproduction, crossover, and nutation, the rlgoritim evolves successive populations in wh she merabers are more "fit."

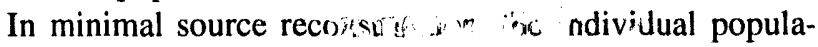
tion members represen a combinations of the potential sources-different subsets of the columns of the $\boldsymbol{A}$ matrix. A column subset is represented by a string of the same length as the number of sources (length of $x$ ) defined over a binary alphabet $(0,1)$. Each element corre- 
sponds to a column (source). A one in a column's position indicates that the corresponding source is active, while a zero indicates that it is not.

The fitness measure used by the genetic algorithm is a function of both compatibility and sparseness. We have used least squares fit error as the measure of compatibility and the number of sources as a measure of sparseness. The general form of the fitness measure to he maximized by the genetic algorithm is

$$
F=\frac{1}{k f_{e}(e)+(1-k) f_{s}(s)}
$$

where $e$ is the least squares fit error, normalized to the range zero to one, $s$ is the ratio of the number of active sources to the rank of $\boldsymbol{A}$, and $k$ is a weighting constant between zero and one. The functions $f_{e}$ and $f_{s}$ are used to shape the response. They are monotonic functions that map the interval $0-1$ onto itself. Specific shaping functions that we have employed include exponentials and $p$ norms.

The genetic algorithm begins with a randomly generated initial population of strings and iteratively evolves successive generations of "better" strings. We have worked with a fixed population size ranging from two to ten times the string length. The algorithm is run until the population converges to steady state.

We have employed two genetic algorithm variants in the evolution of one generation to the next. In the first variant, described by Holland [2], each successive generation is produced by the following operations:

- Evaluate population fitness, order members from high fitness to low fitness, and divide the population into high, medium, and low fitness classes.

- Mate or crossover successive pairs in the high fitness class to produce two offspring per pair and use these offspring to replace the low fitness strings. The high and medium fitness strings carry forward.

- Perform random mutations on the resulting population.

In the second genetic algorithm variant, described by Goldberg [3], each successive generation is produced by the following operations:

- Evaluate population fitness.

- Reproduce an intermediate population by fitnessweighted random selection from the old population.

- Randomly mate or crossover members of the intermediate population and use their offspring to form a new population.
- Perform random mutations on the resulting population.

In both cases the mating is accomplished with uniform cre sover. In this method, one offspring is generated by taking a random selection of its elements from one parent with the remainder coming from the other parent. The second offspring is simply the complementary set of parental elements.

To illustrate the use of genetic algorithms in minimal source reconstruction, we have applied the algorithms to a simulated example derived from the problem of reconstructing neural currents in the human brain from magnetoencephalographic (MEG) measurements of the external neuromagnetic field.

\subsection{Application}

In magnetoencephalography (MEG), the minute magnetic fields $(\sim 100 \mathrm{fT})$ generated by neural activity in the brain are measured at the surface of the head [4,5]. These fields are noninvasively measured using superconducting quantum interference device (SQUID)-based gradiometers. By deducing the sources of these neuromagnetic ficlds-neuromagnetic source reconstruction, a functional image of the brain can be produced that indicates its active regions.

Neuromagnetic source reconstruction is an underdetermined linear inverse problem. Nonzero current distributions can exist that produce no external fields. To address this problem, a priori assumptions must be made about the neural source configuration. A useful configuration model is that of sparse localized sources. This model can be neurologically justified for a large class of experiments, most notably those dealing with evoked responses.

The most common reconstruction approach is a parametric one in which the neural currents are modeled by a small number of current dipoles [6]. The number, locations, orientations, and strengths of these dipoles must then be estimated from the data. This approach has been successful in simple situations in which neural activity is localized in just a few regions. However it is inadequate for analysis of more complex cases [7].

To handle more complex source distributions, a nonparametric reconstruction approach is necessary. The reconstruction problem can be discretized by dividing the brain volume into voxels [8] and representing the neural currents by point current sources (current dipoles) located inside these voxels. The puts the forward problem into the form $y=A x$, where $y$ represents the MEG measurements and $x$ represents the unknown neural source currents. In this application, the number of sources (voxels) can be several orders of magnitude greater than the number of measurements. Appropriate application of anatomi- 
cal constraints, such as limiting the sources to cortex [9], can help, but the result is still a highly underdetermined situation.

Researchers have used minimum norm and weighted minimum norm criteria in reconstructing source configurations $[8,10]$. While this approach is clean and nuathematically tractable, there is no neurological basis for this class of solutions. Minimum norm type solutions tend to be diffuse and are subject to systematic geometrical distortion caused by the rapid fall off of the magnetic field with distance. Minimal source solutions represent a more desirable class of reconstructions $[8,11,12]$. However this approach has been hampered by the difficulty of computing the inverse solutions without resorting to exhaustive search.

\subsection{Example}

To evaluate the potential of algorithms for neuromagnetic source reconstruction, we constructed a simple noiseless simulation example that represents the essence of the problem without imposing undue computational burden. In this example the head is modeled as a sphere with radially symmetric conductivity. In this case, the radial component of the external field produced by a current dipole (point source) can be expressed as

$$
B_{r}=\frac{\vec{M} \bullet \vec{R} \times \vec{L}}{|\vec{R}-\vec{L}|^{3}|\vec{R}|}
$$

where $\vec{M}$ is the dipole moment, $\vec{L}$ is the dipole location, and $\vec{R}$ is the measurement location $[13]$.

Figure 1 shows the geometry and source configuration of our test example. We reconstruct the current flow thru a two-dimensional slice (running through the center head) from the fields observed along its one-dimensional intersection with the head surface. The reconstruction region contains 215 voxels and the measurement set consists of 35 measurements. The measurement locations are denoted by asterisks. The strength of the source voxels are indicated by their shading; white indicates a positive source strength, black indicates a negative source strength, and gray indicates no activity.

Figure 2 shows a minimum norm reconstruction. The solution is diffuse and biased toward the outside of the head. This bias is the result of the inverse squared drop off of the magnetic field with distance. As a result, shallow voxels are unduly favored by the norm metric.

Figure 3 shows a weighted minimum norm reconstruction in which the columns of the $\boldsymbol{A}$ matrix have been scaled to unity to compensate for the magnetic field drop off. The result is less biased, but still quite diffuse. There are broad features in the vicinity of the left and right bipo- lar source pairs. There are no distinguishable features in the vicinity of the deeper central sources.

In Figure 4 we show the result of a minimal source reconstruction computed with a Holland-type algorithm. In this case and in similar simulations the genetic algorithms have been able to pull out underlying sparse solutions.

Overall, genetic algorithms appear to be a promising approach to minimal source reconstruction. However, extensive work still remains to be done in understanding the best algorithm variants to use, in how to set up the myriad of "tweaking" parameters in these algorithms, and in determining performance is the presence of noise and modeling crror.

\section{References}

11] G. Strang. Linear Algebra and its Applications. Third Edition, Harcourt Brace Jovanovich, San Diego, CA, 1988

12) J. Holland. Genetic algorithms. Scientific American, July 1992, pp. 66-72.

13] D. Goldherg. Genetic Algorithms in Search, Optimization \& Machine Learning, Addison-Weslcy, Reading MA, USA. 1989.

14] P. Lewis and J. George. Magnetoencephalography. IEEE Potentials, 9(4):9-13, 1990.

[5] M. Hämäläinen et al. Magnetoencephalography-theory, instrumentation, and applications to noninvasive studies of the working human brain. Reviews of Modern Physics, 65(2):413-497, 1993.

[6] J. Mosher, P. Lewis, and R. Leahy. Multiple dipole modeling and localization from spatio-temporal MEG data. IEEE Trans. Biomedical Engineering, 39(6):541-557, 1992.

[7] J. Mosher, M. Spencer, R. Leahy, and P. Lewis. Error bounds for EEG and MEG dipole source localization. Electroencephalography and clinical Neurophysiology, 86:303-321, 1993.

[8] B. Jeffs, R. Leahy, and M. Singh. An evaluation of methods for neuromagnetic image reconstruction. IEEE Trans. Biomedical Engineering, 34(9):713-723, 1987.

(9) J. George, P. Lewis, D. Rankan, L. Kaplan, and C. Wood. Anatomical constraints for neuromagnetic source models. In Proc. SPIE Medical Imaging V: lmage Physics, 1443:3751, 1991.

[10] A. Ioannides, J. Bolton, and C. Clarke. Continuous probabilistic solutions to the biomagnetic inverse problem. Inverse Problems, 6:523-542, 1990.

[11] B. Jeffs. Maximally Sparse Constrained Optimization for Signal Processing Applications. Ph.D. dissertation, University of Southern California, SIPI Report \#138, 1989.

[12] 1. Gorodnitsky, B. Rao, and J. Gcorge. Source localization in magnetoencephalography using an iterative weighted minimum norm algorithm. In Proc. 26th Asilomar Conf. Signals, Systems \& Computers, Vol. I pp-167-171, Pacific Grove, CA, Nov. 1992.

[13] J. Sarvas. Basic mathematical and electromagnetic concepts of the biomagnetic inverse problem. Phys. Med. Biol, $32(1): 11-22,1987$. 


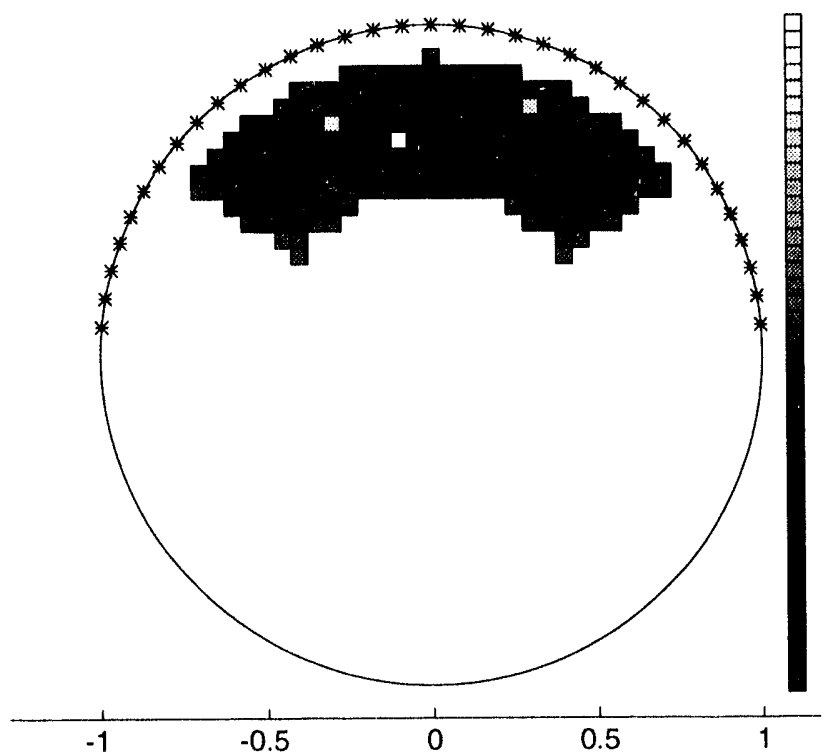

FIGURE 1. Source configuration and example geometry.

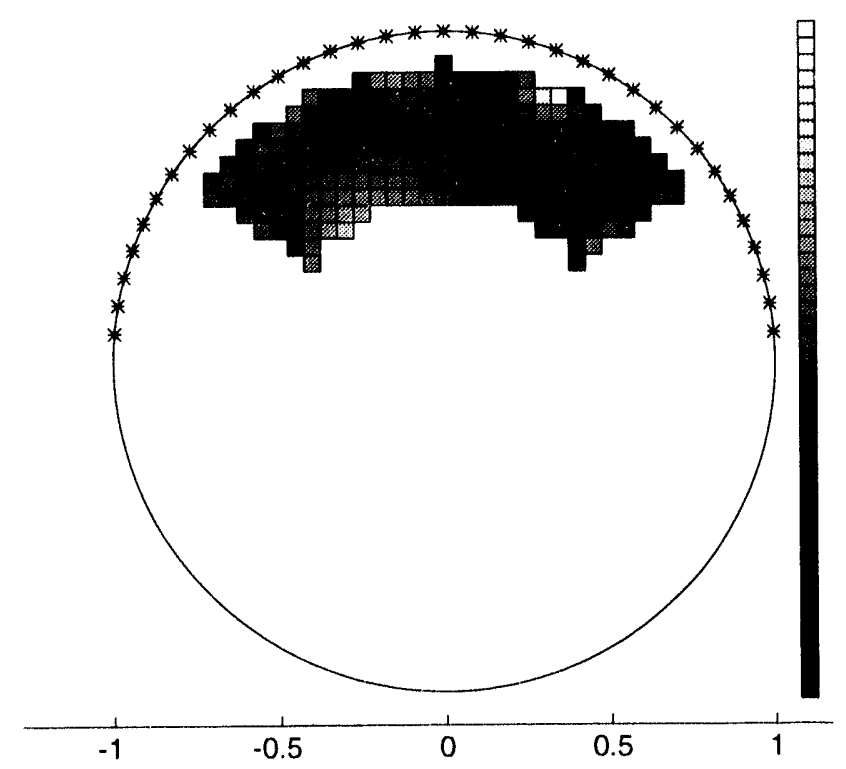

FIGURE 2. Minimum norm reconstruction.

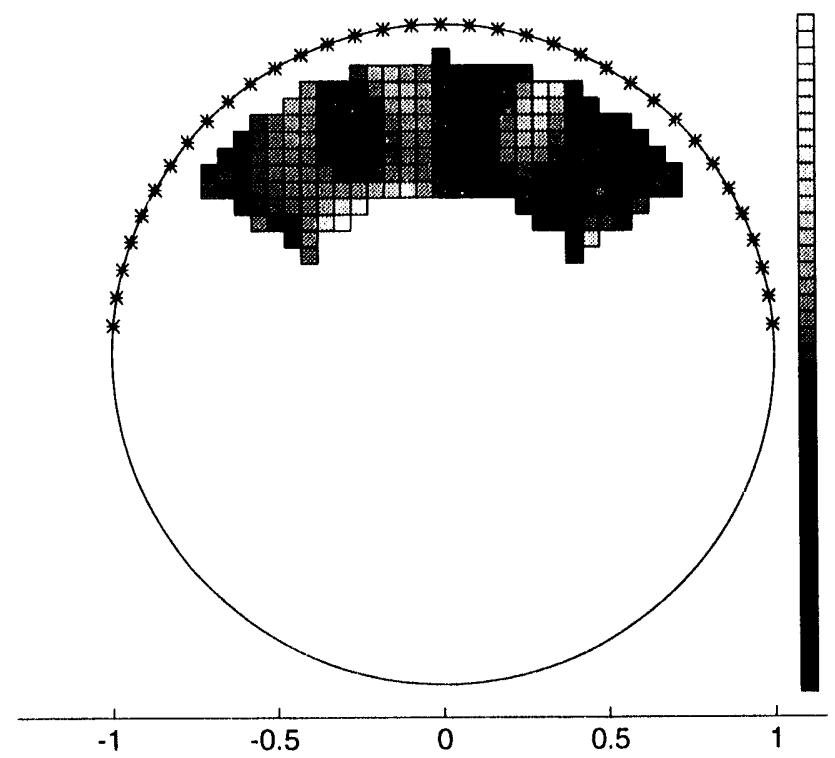

FIGURE 3. Normalized minimum norm reconstruction.

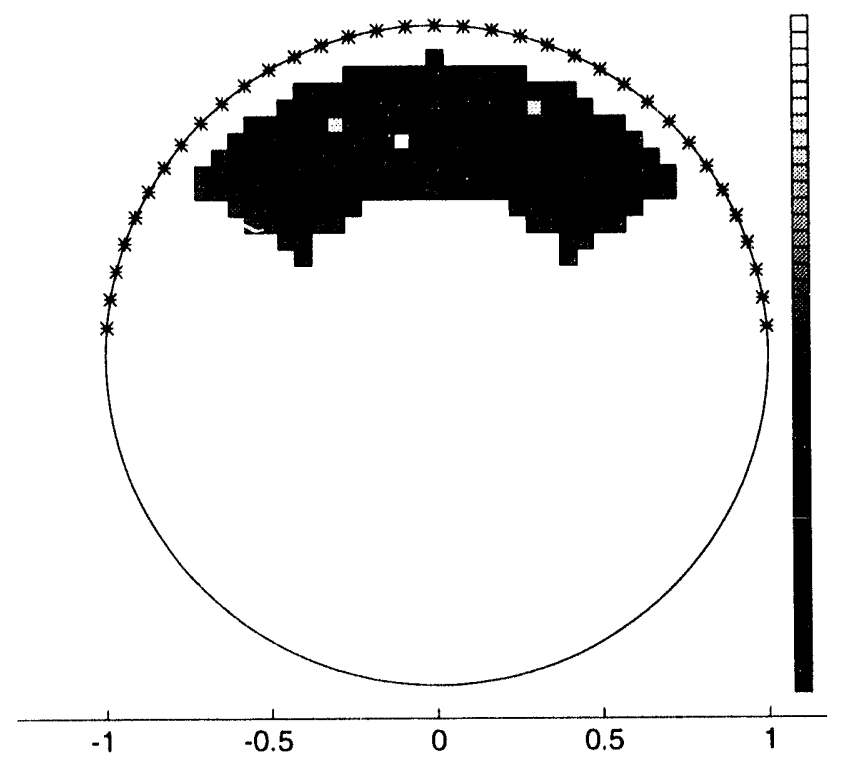

FIGURE 4. Genetic algorithm minimum source reconstruction. 

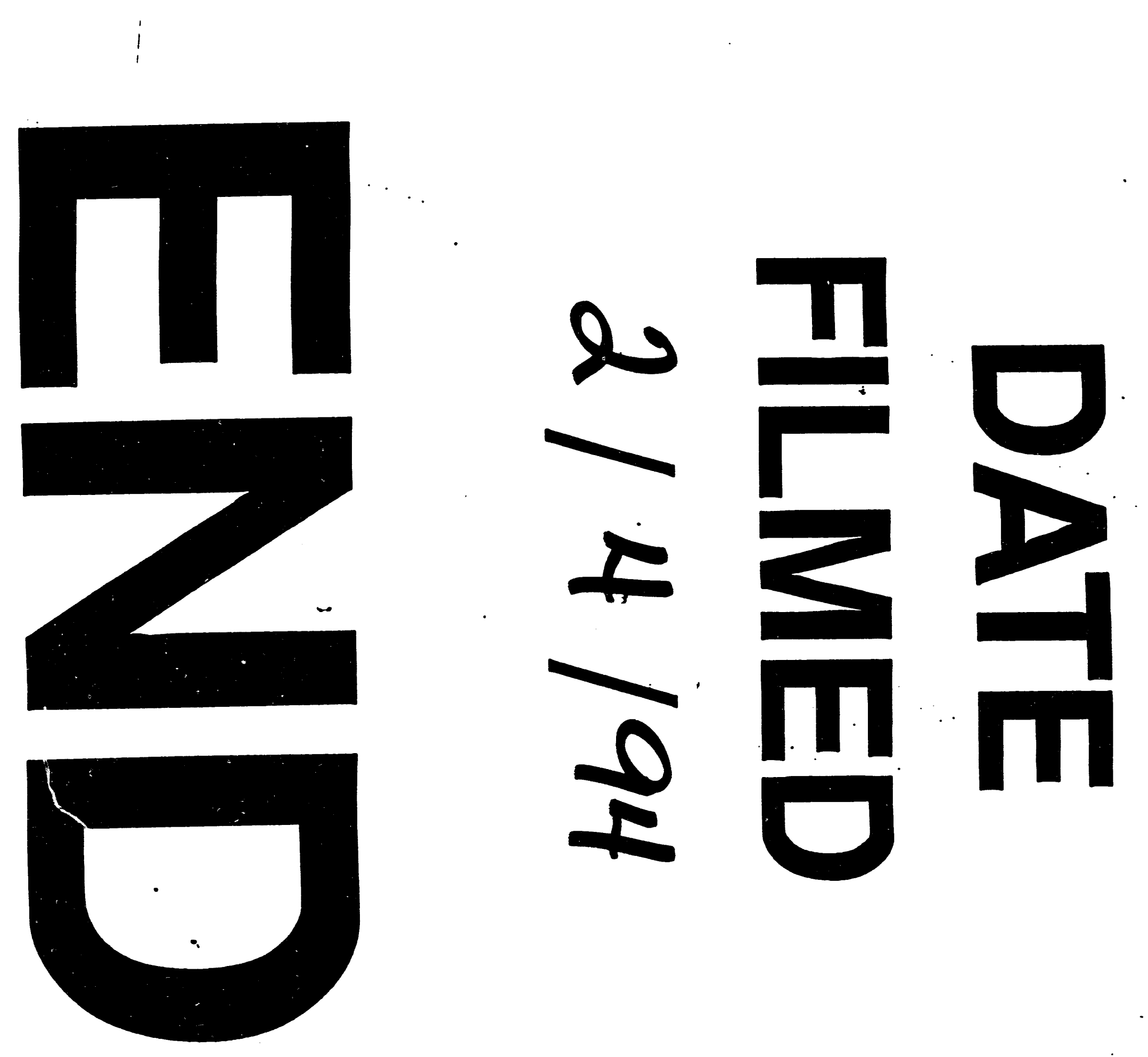
